\title{
Monitoring the $H E R 2$ copy number status in circulating tumor DNA by droplet digital PCR in patients with gastric cancer
}

\author{
Katsutoshi Shoda ${ }^{1,2}$ - Daisuke Ichikawa ${ }^{1}$ Yuji Fujita ${ }^{1,2} \cdot$ Kiyoshi Masuda $^{2}$. \\ Hidekazu Hiramoto $^{1}$ - Junichi Hamada ${ }^{1,2}$ - Tomohiro Arita $^{1}$ • Hirotaka Konishi ${ }^{1}$ • \\ Shuhei Komatsu ${ }^{1}$ - Atsushi Shiozaki ${ }^{1}$ - Naoki Kakihara ${ }^{3} \cdot$ Kazuma Okamoto $^{1}$ •

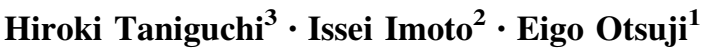

Received: 13 October 2015/ Accepted: 25 January 2016/Published online: 13 February 2016

(C) The International Gastric Cancer Association and The Japanese Gastric Cancer Association 2016

\begin{abstract}
Background We previously demonstrated the potential of circulating tumor DNA (ctDNA) for the amplification of detecting HER2 in patients with gastric cancer (GC). In the present study, we focused on the clinical courses of patients who developed recurrence with GC, and investigated the potential clinical utility of the ddPCR-based HER2 copy number $(\mathrm{CN})$ as a marker for the temporal and/or spatial heterogeneities of GC during treatment progress.

Method We enrolled 30 healthy volunteers and 60 patients with GC who underwent surgery, including 17 patients who developed recurrence. Using ribonuclease $\mathrm{P}$ RNA component $\mathrm{H} 1(R P P H I)$ as a reference gene, plasma $H E R 2$ to RPPH1 ratios (the HER2 ratio) were determined using ddPCR.

Results The preoperative plasma HER2 ratio correlated with the tumor HER2 status $(p<0.001)$, and sensitivity and specificity were 0.733 and 0.933 , respectively.
\end{abstract}

Electronic supplementary material The online version of this article (doi:10.1007/s10120-016-0599-z) contains supplementary material, which is available to authorized users.

Daisuke Ichikawa
ichikawa@koto.kpu-m.ac.jp
Issei Imoto
issehgen@tokushima-u.ac.jp

Division of Digestive Surgery, Department of Surgery, Kyoto Prefectural University of Medicine, 465 Kawaramachi Hirokoji Kajii-cho, Kamigyo-ku, Kyoto 602-8566, Japan

2 Department of Human Genetics, Institute of Health Biosciences, The University of Tokushima Graduate School, 3-18-15 Kuramoto-cho, Tokushima 770-8503, Japan

3 Department of Surgery, Kyoto Second Red Cross Hospital, 355-5 Haruobi-cho, Kamigyo-ku, Kyoto 602-8026, Japan
Analyses of plasma samples during the postoperative follow-up periods revealed that high plasma HER2 ratios were detected at the time of recurrence in 7 of 13 cases, which were diagnosed as being HER2 negative at the time of surgery. These results were supported by continuously increasing $H E R 2$ ratios thereafter with the progression of recurrent cancer.

Conclusion The plasma HER2 ratio determined by ddPCR is a repeatable and noninvasive approach for realtime evaluations of the HER2 status to monitor the effects of treatments for patients with HER2-positive GC and enable treatment options for patients with HER2-negative GC but positive conversion of the HER2 status after recurrence.

Keywords Gastric cancer $\cdot$ HER2 $\cdot$ DdPCR $\cdot$ ctDNA . Liquid biopsy

\section{Introduction}

The amplification and overexpression of human epidermal growth factor receptor 2 (HER2), which contributes to cancer progression [1], have been reported in approximately $20 \%$ of patients with advanced gastric cancer (GC) and were shown to be associated with a more aggressive phenotype and poorer survival in a meta-analysis [2]. A phase III trial of trastuzumab for patients with HER2positive advanced GC (the ToGA trial) demonstrated that the addition of trastuzumab to chemotherapy was significantly beneficial in terms of overall survival versus chemotherapy alone [3]. Based on these findings, the administration of trastuzumab to patients with recurrent GC was recommended by the European Medicines Agency and the Japanese Gastric Cancer Association (JGCA) in 
2010 and 2011, respectively. They noted that trastuzumab should be administered to patients with HER2-positive tumors as routinely defined by immunohistochemistry (IHC) $3+$ or IHC $2+$ with a positive fluorescent in situ hybridization (FISH) result because the recurrent tumors that developed in these patients were likely to be HER2 positive. However, the response rate to trastuzumab remains insufficient, even in these eligible patients [3]. Furthermore, the number of patients eligible for trastuzumab treatments is limited. Because the distribution of HER2-expressing cells is known to be heterogeneous in GC [4], HER-2 positivity in resected tumors may provide insufficient information for determining eligibility for trastuzumab treatments because of the temporal and spatial intratumoral and intertumoral heterogeneities of this disease. Recurrent tumors from HER2-positive cases may contain HER2-negative tumor cells as a major population, whereas HER2 amplification/overexpression may be acquired or HER2-positive cells existing as a minor population in resected tumors may be selected during treatment progress in HER2-negative cases. Liquid biopsy has recently attracted the interest of clinicians as a possible approach that may overcome the influence of heterogeneity $[5,6]$, with a number of studies being conducted to investigate intratumoral heterogeneity [7-9]. The potential of circulating tumor DNA (ctDNA) as the source of a template in minimally invasive diagnostics is becoming increasingly apparent for monitoring the status of tumors with temporal and spatial heterogeneities [10-12].

The detection and quantitation of specific nucleic acid sequences using a polymerase chain reaction (PCR) is fundamental to a large body of research and a growing number of molecular diagnostic tests, including HER2 amplification [13]. We previously demonstrated the possible clinical utility and usefulness of liquid biopsies using real-time quantitative PCR (rqPCR), a second-generation PCR-based quantification method in patients with GC [9]. However, quantitative information in rqPCR is obtained from the cycle threshold (CT). Imperfect amplification efficiencies affect CT values, which, in turn, limit the accuracy of this technique for absolute quantitation, and the $Z$ score to separate positive and negative results needs to be determined for each experimental set. One method showing promise for improving the limit of detection for nucleic acid quantification is digital PCR (dPCR), with a number of studies highlighting the superior accuracy of dPCR for copy number $(\mathrm{CN})$ analyses $[14,15]$, including HER2 amplification in circulating ctDNA in breast cancer (BC) and GC [16, 17]. These studies demonstrated the potential of HER2 amplification detected in preoperative ctDNA by dPCR to precisely estimate the HER2 status of tumors or prognosis of patients.
In the present study, we used digital droplet PCR (ddPCR) to determine the HER2 CN status in ctDNA samples collected from GC patients, and evaluated the potential clinical utility of the ddPCR-based $H E R 2 \mathrm{CN}$ as a marker for the temporal and/or spatial heterogeneities of GC by monitoring the dynamics of the plasma HER2 CN status during treatment progress.

\section{Materials and methods}

\section{Patients and samples}

Our study protocol was approved by the local Ethics Committee and conducted in accordance with the Declaration of Helsinki. All subjects gave their written informed consent before participating. Samples were blinded for analyses, and patients understood that the results would not be made available to them. The study cohort (Table 1) included 60 patients with GC who underwent surgery and had tissue HER2 status determinations between January 2009 and December 2014 at the Kyoto Prefectural University of Medicine Hospital. Preoperative plasma samples were collected from all patients. Of these, 36 patients were also included in our previous study [6]. Control plasma samples were obtained from 30 healthy adult volunteers by standard antecubital venous puncture.

Patient demographic data and details regarding tumor recurrence and subsequent management were recorded. Computed tomography (CT) imaging was performed and reviewed in a blinded manner to document treatment responses according to the Response Evaluation Criteria in Solid Tumors (RECIST), version 1.1 [18]. The pathological classification of tumors was determined according to the UICC classification [19]. The tissue HER2 status was determined by FFPE using routine IHC and FISH methods. Following the American Society of Clinical Oncologists/ College of American Pathologists guideline recommendations, IHC 0 and IHC $1+$ were both considered to be HER2 negative, whereas IHC $3+$ was defined as HER2 positive. IHC 2+ was considered equivocal; a case was considered to be HER2 positive if it was IHC $2+$ plus FISH with a threshold ratio $>2.0$ between the HER2 gene $\mathrm{CN}$ and chromosome 17 centromere (HER2:CEP17). Trastuzumab therapy was administered to HER2-positive cases with recurrence. As traditional tumor markers, carcinoembryonic antigen (CEA) and carbohydrate antigen (CA) were measured in each case, with cutoff values of $5.0 \mathrm{ng} / \mathrm{ml}$ and $37 \mathrm{U} / \mathrm{ml}$, respectively. A DNA sample (POS2000) obtained from a GC tumor was used as a positive control, and its $H E R 2 \mathrm{CN}$ was confirmed in our previous study. 
Table 1 Clinicopathological features of 60 gastric cancer (GC) patients analyzed for preoperative plasma droplet digital PCR (ddPCR) HER2 ratios

\begin{tabular}{|c|c|c|c|c|c|c|c|c|c|c|c|}
\hline \multirow[t]{3}{*}{ Features } & \multirow[t]{3}{*}{$n$} & \multicolumn{5}{|c|}{ 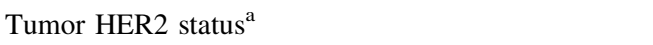 } & \multicolumn{5}{|c|}{ Plasma $H E R 2$ amplification $^{\mathrm{b}}$} \\
\hline & & \multicolumn{2}{|c|}{ Negative } & \multicolumn{2}{|c|}{ Positive } & \multirow[t]{2}{*}{$p^{\mathrm{c}}$} & \multicolumn{2}{|c|}{ Absent } & \multicolumn{2}{|c|}{ Present } & \multirow[t]{2}{*}{$P^{\mathrm{c}}$} \\
\hline & & $n$ & $\%$ & $n$ & $\%$ & & $n$ & $\%$ & $n$ & $\%$ & \\
\hline Total & 60 & 45 & $(75.0)$ & 15 & $(25.0)$ & & 46 & $(76.7)$ & 14 & $(23.3)$ & \\
\hline \multicolumn{12}{|l|}{ Gender } \\
\hline Male & 44 & 33 & $(75.0)$ & 11 & $(25.0)$ & \multirow[t]{2}{*}{1.0000} & 32 & $(72.7)$ & 12 & $(27.3)$ & \multirow[t]{2}{*}{0.2099} \\
\hline Female & 16 & 12 & $(75.0)$ & 4 & $(25.0)$ & & 14 & $(87.5)$ & 2 & $(12.5)$ & \\
\hline \multicolumn{12}{|l|}{ Age (years) } \\
\hline$<70$ & 38 & 30 & $(78.9)$ & 8 & $(21.1)$ & \multirow[t]{2}{*}{0.3580} & 30 & $(78.9)$ & 8 & $(21.1)$ & \multirow[t]{2}{*}{0.5857} \\
\hline$\geq 70$ & 22 & 15 & $(68.2)$ & 7 & $(31.8)$ & & 16 & $(72.7)$ & 6 & $(27.3)$ & \\
\hline \multicolumn{12}{|l|}{ Location } \\
\hline Upper & 21 & 14 & $(66.7)$ & 7 & $(33.3)$ & \multirow[t]{2}{*}{0.2800} & 13 & $(61.9)$ & 8 & $(38.1)$ & \multirow[t]{2}{*}{0.0514} \\
\hline Middle or lower & 39 & 31 & $(79.5)$ & 8 & $(20.5)$ & & 33 & $(84.6)$ & 6 & $(15.4)$ & \\
\hline \multicolumn{12}{|c|}{ Histopathological predominant ${ }^{\mathrm{d}}$} \\
\hline Differentiated & 21 & 12 & $(57.1)$ & 9 & $(42.9)$ & \multirow[t]{2}{*}{0.0212} & 12 & $(57.1)$ & 9 & $(42.9)$ & \multirow[t]{2}{*}{0.0100} \\
\hline Undifferentiated & 39 & 33 & $(84.6)$ & 6 & $(15.4)$ & & 34 & $(87.2)$ & 5 & $(12.8)$ & \\
\hline Size & & & & & & & & & & & \\
\hline$<90$ & 32 & 26 & $(81.3)$ & 6 & $(18.8)$ & 0.2317 & 28 & $(87.5)$ & 4 & $(12.5)$ & 0.0323 \\
\hline$\geq 90$ & 28 & 19 & $(67.9)$ & 9 & $(32.1)$ & & 18 & $(64.3)$ & 10 & $(35.7)$ & \\
\hline Lymphatic invasior & & & & & & & & & & & \\
\hline Negative & 14 & 12 & $(85.7)$ & 2 & $(14.3)$ & 0.2693 & 12 & $(85.7)$ & 2 & $(14.3)$ & 0.3415 \\
\hline Positive & 46 & 33 & (71.7) & 13 & (28.3) & & 34 & (73.9) & 12 & (26.1) & \\
\hline Venous invasion & & & & & & & & & & & \\
\hline Negative & 35 & 28 & $(80.0)$ & 7 & $(20.0)$ & 0.2924 & 27 & $(77.1)$ & 8 & (22.9) & 0.9179 \\
\hline Positive & 25 & 17 & $(68.0)$ & 8 & $(32.0)$ & & 19 & $(76.0)$ & 6 & $(24.0)$ & \\
\hline Depth of tumor inv & & & & & & & & & & & \\
\hline $\mathrm{pT} 1 / 2 / 3$ & 25 & 20 & $(80.0)$ & 5 & $(20.0)$ & 0.4458 & 20 & $(80.0)$ & 5 & $(20.0)$ & 0.6038 \\
\hline pT4 & 35 & 25 & (71.4) & 10 & $(28.6)$ & & 26 & (74.3) & 9 & $(25.7)$ & \\
\hline N stage $e^{e}$ & & & & & & & & & & & \\
\hline pNO & 10 & 8 & $(80.0)$ & 2 & $(20.0)$ & 0.6833 & 8 & $(80.0)$ & 2 & $(20.0)$ & 0.7818 \\
\hline $\mathrm{pN} 1-3$ & 50 & 37 & $(74.0)$ & 13 & $(26.0)$ & & 38 & (76.0) & 12 & $(24.0)$ & \\
\hline pStage $^{\mathrm{e}}$ & & & & & & & & & & & \\
\hline $\mathrm{pI} / \mathrm{pII}$ & 13 & 11 & $(84.6)$ & 2 & $(15.4)$ & 0.3468 & 11 & (84.6) & 2 & (15.4) & 0.4279 \\
\hline pIII & 47 & 34 & $(72.3)$ & 13 & $(27.7)$ & & 35 & $(74.5)$ & 12 & $(25.5)$ & \\
\hline HER2 status by IH & & & & & & & & & & & \\
\hline 0 & 24 & 24 & $(100.0)$ & 0 & $(0.0)$ & $<0.0001$ & 22 & $(91.7)$ & 2 & (8.3) & $<0.0001$ \\
\hline 1 & 16 & 16 & $(100.0)$ & 0 & $(0.0)$ & & 15 & (93.8) & 1 & (6.3) & \\
\hline 2 & 7 & 5 & $(71.4)$ & 2 & $(28.6)$ & & 6 & $(85.7)$ & 1 & $(14.3)$ & \\
\hline 3 & 13 & 0 & $(0.0)$ & 13 & (100.0) & & 3 & $(23.1)$ & 10 & $(76.9)$ & \\
\hline Tumor HER2 statu & & & & & & & & & & & \\
\hline Negative & 45 & - & & - & & & 42 & $(93.3)$ & 3 & $(6.7)$ & $<0.0001$ \\
\hline Positive & 15 & - & & - & & & 4 & (26.7) & 11 & (73.3) & \\
\hline
\end{tabular}

${ }^{a}$ Tumor HER2 status was determined by an immunohistochemistry (IHC) score of 3 or IHC score of 2 with a positive FISH score determined as described in the "Materials and methods" section

b Plasma HER2 amplification was determined by the plasma HER2 ratio using ddPCR with 2.10 as a cutoff value

${ }^{c} p$ values are from the $\chi 2$ or Fisher's exact test and were significant at less than 0.05 . Significant values are shown in boldface

d "Differentiated" corresponds to tubular adenocarcinoma or papillary adenocarcinoma, and "undifferentiated" corresponds to poorly differentiated adenocarcinoma, signet-ring carcinoma, or mucinous adenocarcinoma

e Disease stage was defined in accordance with the International Union Against Cancer (UICC) 7th tumor-lymph node-metastases (TNM) classification using surgical pathological findings 


\section{Preparation and DNA isolation from plasma samples}

A 7-ml EDTA blood sample was obtained from each patient before surgery and from 30 healthy volunteers. Samples were also collected from 21 and 17 of the 60 patients 1 month after surgery and at recurrence, respectively. In those 21 cases, samples were corrected repeatedly after surgery in 6 cases with recurrence and 1 case without recurrence. Plasma was immediately separated from the cellular fraction using a three-spin protocol as described elsewhere [20], and then stored at $-80{ }^{\circ} \mathrm{C}$ for further processing. Circulating ctDNA was isolated from $2 \mathrm{ml}$ of a plasma sample with a QIAamp Circulating Nucleatic Acid kit (Qiagen, Hilden, Germany). The mean ctDNA amount per $1 \mathrm{ml}$ plasma was $13.61 \mathrm{ng}$.

\section{HER2 CN analysis using ddPCR}

Using ribonuclease P RNA component $H 1$ (RPPHl) as an internal control to assess the $\mathrm{CN}$ of the HER2 gene [6], plasma HER2-to-RPPH1 ratios (the HER2 ratio) were determined using ddPCR. Each sample was partitioned into 20,000 droplets, with target and control (background) DNA being randomly, but uniformly distributed among the droplets. As ctDNA samples are highly fragmented, product sizes for ddPCR reaction of HER 2 and $R P P H 1$ are set to be $90 \mathrm{bp}$ and $92 \mathrm{bp}$, respectively. The following primers were used for ddPCR: HER2 forward (5'-CTCATCGCTC ACAACCAAGT- $\left.3^{\prime}\right), \quad H E R 2$ reverse (5'-CAGGGCA TAGTTGTCCTCAA- ${ }^{\prime}$ ), the FAM probe for HER2 (5'-FA M-TGTGCGAGGCACCCAGCTCT-3'), RPPHI forward (5'-GTCAGACTGGGCAGGAGATG-3'), RPPHI reverse (5'-TGGCCGTGAGTCTGTTCC- $3^{\prime}$ ), and the HEX probe for RPPHI (5'-HEX-TGCCTCCTTTGCCGGAGCTT- $\left.3^{\prime}\right)$. Reactions were performed in $20-\mu l$ reaction volumes that consisted of extracted DNA $(4 \mu \mathrm{l}), 2 \times$ ddPCR supermix for the probe $(10 \mu \mathrm{l}), 20 \mu \mathrm{M} H E R 2$ forward primer $(0.9 \mu \mathrm{l}), 20 \mu \mathrm{M}$ HER 2 reverse primer $(0.9 \mu \mathrm{l})$, the HER 2 FAM probe $(0.25 \mu \mathrm{l}), 20 \mu \mathrm{M} R P P H 1$ forward primer $(0.9 \mu \mathrm{l}), 20 \mu \mathrm{M} R P P H 1$ reverse primer $(0.9 \mu \mathrm{l})$, the $R P P H 1$ HEX probe $(0.25 \mu \mathrm{l})$, and deionized distilled water $(1.9 \mu \mathrm{l})$. Emulsified PCR reactions were run in a 96-well plate on a LifePro Thermal Cycler (BIOER, Hangzhou, China). After a suitable annealing temperature had been determined for distinguishing between positive and negative droplets (Fig. S1a), the plates were incubated at $95{ }^{\circ} \mathrm{C}$ for $10 \mathrm{~min}$, followed by 40 cycles of $94{ }^{\circ} \mathrm{C}$ for $30 \mathrm{~s}, 57^{\circ} \mathrm{C}$ for $60 \mathrm{~s}$, and a 10-min incubation at $98{ }^{\circ} \mathrm{C}$. The plates were read on a Bio-Rad QX200 droplet reader (Bio-Rad, Hercules, CA, USA) using the QuantaSoft v1.4.0 software (Bio-Rad) to assess the number of droplets positive for HER2 and RPPHI.

\section{HER2 CN analysis using rqPCR}

A quantitative analysis of $H E R 2$ amplification using rqPCR was performed on the cell-free DNA (cfDNA) samples of 30 healthy volunteers as previously described elsewhere [6].

\section{Statistical analysis}

Spearman's correlation coefficients were determined to assess the relationships among $\mathrm{CN}$ data determined by different methods. Nonparametric tests were used for subgroup comparisons (Wilcoxon rank-sum test) and for comparisons between paired samples in each subgroup (Wilcoxon signed-rank test). The $\chi^{2}$ or Fisher's exact test was used to assess the relationships between plasma HER2 ratio results and clinicopathological factors. All statistical tests, except for paired tests, were two sided. Significance was accepted at $p$ values $<0.05$.

\section{Results}

\section{Setting a cutoff value for $\mathrm{ddPCR}$}

We evaluated the reproducibility of ddPCR for evaluating the HER2 CN. Using ddPCR, CNs for HER2, and an internal control, RPPHI of the positive control DNA sample (POS2000) obtained from the GC tumor, the HER2 $\mathrm{CN}$ of which was confirmed beforehand, were found to be 1962 and 69.5 in 10 ng DNA, respectively. The range of the reproducibility of ddPCR in a small amount of DNA was determined using serial dilutions starting from $10 \mathrm{ng}$ of the POS2000 sample (Fig. S1b, S1c). The CNs of all cfDNA samples used in the present study were within this range in the ddPCR analysis.

To set a cutoff value for plasma $H E R 2$ to $R P P H 1$ ratios (the HER2 ratio), we determined the HER2 ratio of plasma cfDNA (plasma HER2 ratio) obtained from 30 healthy volunteers using two methods; ddPCR and rqPCR, and compared the results obtained (Fig. 1a). The plasma HER2 ratios determined by these two methods were correlated (Fig. 1a, right). The standard deviation (SD) of the plasma HER2 ratio determined by ddPCR was smaller than that determined by rqPCR (Fig. 1a, left), and the $95 \%$ confidence intervals (CI) of ddPCR and rqPCR were 0.945-1.052 and 0.923-1.077, respectively. Because the means and SDs of the plasma HER2 ratios determined by ddPCR in the normal cohort were 1.619 and 0.230 , respectively, we set the cutoff value for the plasma $H E R 2$ ratio at 2.1 for plasma $H E R 2$ ratios based on the mean plus 2 SD of the normal cohort (Fig. 1b). All results from the normal cohort were less than this cutoff value. 
Fig. 1 Potential of digital droplet PCR (ddPCR) as a method to evaluate the HER2 copy number $(\mathrm{CN})$ status in cell-free DNA (cfDNA) samples. a Comparison between ddPCR and real-time quantitative PCR (rqPCR) in cfDNA with 30 healthy volunteers. Left: Standard deviations (SD) of ddPCR and rqPCR were 0.1432 and 0.2057 , respectively, when both means were set to 1.00 (relative quantity, RQ). Right: The results obtained using both methods were consistent ( $\rho=0.3939, p=0.0313$; Spearman's analysis). b Plasma HER2 ratios for healthy volunteers, gastric cancer (GC) patients with HER2-negative tumors, and those with HER2positive tumors. The cutoff value for the plasma $H E R 2$ ratio test to detect HER2

amplification in GC was determined using the mean plus 2 SD of healthy volunteers. Plasma HER2 ratios were significantly higher in HER2positive patients than in HER2negative patients $(p=0.0006$; Wilcoxon rank-sum test). The sensitivity, specificity, positive predicting value (PPV), and negative predicting value (NPV) of the cutoff value to discriminate patients with HER2-positive from those with HER2-negative GC tumors were $0.7333,0.9333,0.7857$, and 0.9130 , respectively. c Comparison of plasma HER2 ratios before and 1 month after surgery in $8 \mathrm{GC}$ patients with plasma $H E R 2$ amplification before surgery (left) and 13 patients without plasma HER2 amplification before surgery (right). Note that decreases in plasma HER2 ratios were observed in most patients with plasma HER2 amplification
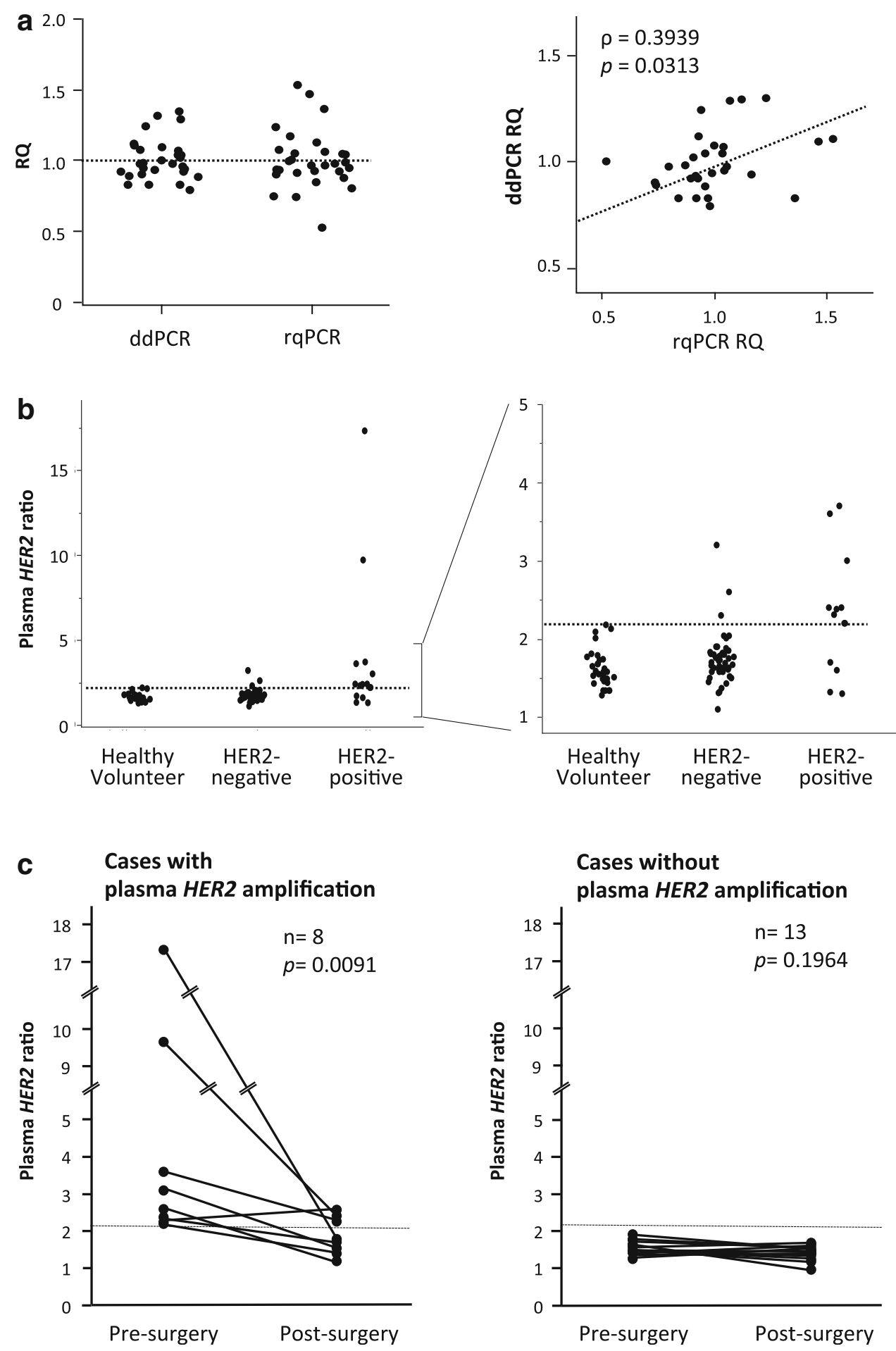

\section{Sensitivity and specificity of the plasma $H E R 2$ ratio for detecting the tumor HER2 status in GC}

We measured plasma HER2 ratios in 60 patients with HER2-negative or HER2-positive GC. The means and SDs of the plasma HER2 ratios obtained were 1.790 and 0.383 in HER2-negative patients and 4.912 and 5.284 in HER2- positive patients, respectively. Plasma HER2 ratios were significantly higher in HER2-positive patients than in HER2-negative patients $(p=0.0006$; Wilcoxon rank-sum test) (Fig. 1b). The sensitivity and specificity of the cutoff value for plasma HER2 ratios (2.1) were 0.7333 and 0.9333 , respectively, and the positive predicting value (PPV) and negative predicting value (NPV) were 0.7857 
and 0.9130 , respectively. The same cutoff value (2.1) was obtained when we determined the best discriminating level of the HER2 ratio to separate HER2-positive patients from HER2-negative patients by generating a receiver operating characteristic curve and calculating the area under the curve. In 36 cases with plasma HER 2 ratios determined by ddPCR in this study and rqPCR in our previous study [6], sensitivity and specificity were higher in the ddPCR-based method than in the rqPCR-based method (sensitivity and specificity were 0.700 and 1.000 in ddPCR, and 0.400 and 0.962 in rqPCR, respectively). Because an absolute $\mathrm{CN}$ is obtainable in digital PCR, which results in a more objective evaluation without sharing the calibrator sample between experiments and laboratories [21], these results may reflect the greater accuracy and reproducibility of ddPCR over rqPCR.

Plasma HER2 ratios were compared between pre- and paired postoperative samples in 21 cases. These ratios were significant lower in postoperative samples than in preoperative samples in patients with plasma HER2 amplification before surgery ( $n=8 ; p=0.0091$; Wilcoxon signedrank test) (Fig. 1c), which also supports the reproducibility of the ddPCR-based method. Three cases with plasma HER2 ratio higher than the cutoff value even 1 month after surgery showed early recurrence 3,3 , and 5 months after surgery, respectively.

\section{Relationships between clinicopathological parameters and plasma $H E R 2$ ratios determined by $\operatorname{ddPCR}$}

The relationships between clinicopathological characteristics and HER2 statuses determined by the two methods, routine methods, and the plasma HER2 ratio in all 60 patients with GC are summarized in Table 1 . The presence of plasma HER2 amplification was associated with the differentiated histopathological type as the routine methodbased tumor HER2 status. However, only plasma HER2 amplification positively correlated with tumor size (Table 2, Fig. 2). Plasma HER2 ratios in HER2-positive cases correlated with tumor sizes in Spearman's analysis ( $\rho=0.6047, p=0.0169$; Fig. 2). Plasma HER2 ratios also correlated with the FISH score (HER2:CEP17; $\rho=0.5437, p=0.0132, n=20$; Fig. S2). Because four HER2-positive cases with negative plasma HER2 amplification had smaller tumor sizes, the size of the HER2 amplification-positive tumor cell population and/or $\mathrm{CN}$ in each cell may have affected the sensitivity to detect plasma

Table 2 Dynamics of plasma HER2 ratios in recurrent GC patients with adjuvant chemotherapy

\begin{tabular}{|c|c|c|c|c|c|c|c|c|c|c|c|c|}
\hline \multirow[t]{2}{*}{ Case ID } & \multirow[t]{2}{*}{ Age (years) } & \multirow[t]{2}{*}{ Gender } & \multirow[t]{2}{*}{ Stage } & \multirow[t]{2}{*}{ Type of recurrence } & \multicolumn{3}{|c|}{ Tissue Her2 status ${ }^{\mathrm{a}}$} & \multicolumn{4}{|c|}{ cfDNA $H E R 2$ amplification $^{\text {b }}$} & \multirow{2}{*}{$\begin{array}{l}\text { Trastuzumab } \\
\text { treatment }^{\mathrm{c}}\end{array}$} \\
\hline & & & & & HER2 & $\mathrm{IHC}$ & FISH & Pre-surger & & Recurrenc & & \\
\hline 1 & 63 & $\mathrm{~F}$ & IIIC & Lymphatic & Negative & 2 & 1.2 & Negative & 1.6 & Positive & 2.2 & - \\
\hline 2 & 63 & M & IIIC & Lymphatic & Negative & 1 & - & Negative & 1.3 & Positive & 2.2 & - \\
\hline 3 & 63 & M & IIIA & Hematogenous & Negative & 2 & 1.5 & Negative & 1.6 & Positive & 2.1 & - \\
\hline 4 & 62 & M & IIIB & Peritoneal & Negative & 0 & - & Negative & 1.4 & Positive & 2.9 & - \\
\hline 5 & 70 & M & IA & Hematogenous & Negative & 1 & - & Negative & 1.9 & Positive & 2.1 & - \\
\hline 6 & 53 & $\mathrm{~F}$ & IIIC & Peritoneal & Negative & 1 & - & Negative & 1.8 & Positive & 3.3 & - \\
\hline 7 & 75 & M & IIIA & Peritoneal & Negative & 0 & - & Negative & 1.7 & Positive & 2.1 & - \\
\hline 8 & 59 & $\mathrm{~F}$ & IIIC & Peritoneal & Negative & 0 & - & Negative & 1.6 & Negative & 1.5 & - \\
\hline 9 & 67 & M & II & Peritoneal & Negative & 0 & - & Negative & 1.8 & Negative & 1.8 & - \\
\hline 10 & 73 & M & IA & Hematogenous & Negative & 0 & - & Negative & 2.0 & Negative & 1.7 & - \\
\hline 11 & 72 & M & IIIC & Peritoneal & Negative & 1 & - & Negative & 1.8 & Negative & 1.0 & - \\
\hline 12 & 53 & $\mathrm{~F}$ & IIIC & Peritoneal & Negative & 1 & - & Negative & 1.1 & Negative & 1.7 & - \\
\hline 13 & 61 & M & IIIC & Lymphatic & Negative & 1 & - & Positive & 3.2 & Negative & 1.7 & - \\
\hline 14 & 82 & M & IIIB & Lymphatic & Positive & 3 & - & Positive & 3.7 & Positive & 5.8 & PR \\
\hline 15 & 65 & $\mathrm{~F}$ & IIIB & Hematogenous & Positive & 3 & 2.3 & Negative & 1.7 & Positive & 2.4 & PR \\
\hline 16 & 72 & $\mathrm{~F}$ & IIIC & Lymphatic & Positive & 3 & 4.5 & Positive & 2.4 & Positive & 6.4 & PR \\
\hline 17 & 61 & M & IIIA & Peritoneal & Positive & 2 & 2.3 & Negative & 1.3 & Negative & 1.3 & SD \\
\hline
\end{tabular}

Amplification-positive results are shown in boldface

$P R$ partial response, $S D$ stable disease

${ }^{a}$ Tumor HER2 statuses were determined by an immunohistochemistry (IHC) score of 3 or an IHC score of 2 with a positive FISH score determined as described in the "Materials and methods" section

b cfDNA HER2 amplification was determined by the ddPCR plasma HER2 ratio test with 2.10 as a cutoff value

c Trastuzumab therapy for patients with HER2-positive gastric cancer was administrated on the basis of the European Medicines Agency criteria 


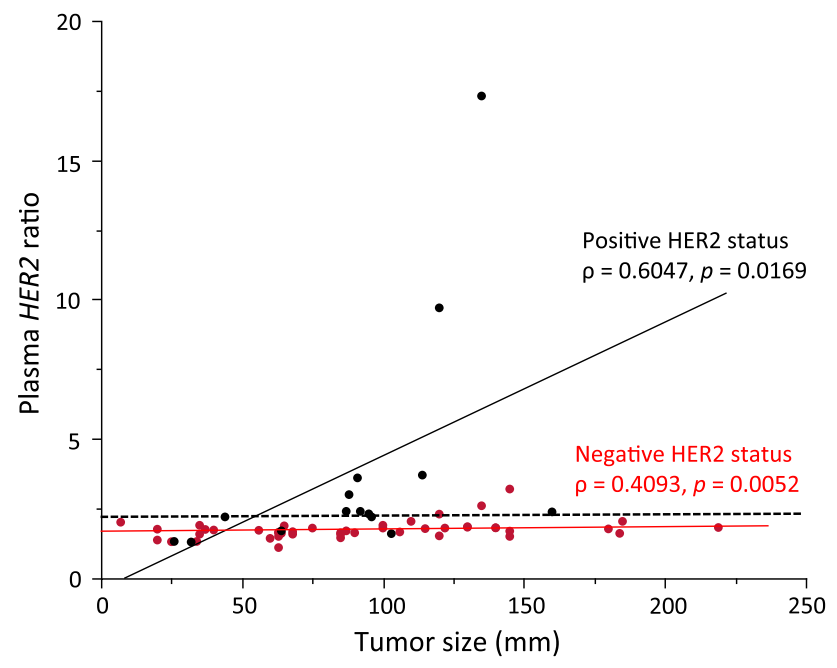

Fig. 2 Relationship between tumor size and the plasma HER2 ratio in patients with GC. Black and red dots represent values for each patient with HER2-positive and HER-2-negative GC, respectively. Plasma HER2 ratios correlated with tumor sizes in patients with HER2-positive and HER2-negative GC $(p=0.0169$, and 0.0052, respectively; Spearman's correlation)

HER2 amplification in ctDNA, suggesting a possible reflection on the amount of HER2-positive tumor cells by the plasma HER2 ratio, even with the presence of heterogeneity in GC.

In HER2-negative cases, plasma HER2 ratios also correlated with tumor sizes in Spearman's analysis ( $\rho=0.4093, p=0.0052$; Fig. 2$)$. All three HER2-negative cases with positive plasma HER2 amplification had larger tumor sizes. These results suggest that HER2-positive cells exist in tumors as a minor population and the routine method to detect the tumor HER2 expression/amplification status underestimates or overlooks these cells. Although it is possible that cells in minor populations may be completely resected in surgery, some patients eligible for trastuzumab therapy may be incorrectly assessed by the routine method.

\section{Dynamics of the plasma $H E R 2$ ratio during therapeutic courses}

We measured the plasma $H E R 2$ ratio in $17 \mathrm{GC}$ patients at the time of recurrence: 13 and 4 cases were HER 2 negative and HER 2 positive in primary GC tumors, respectively. In 6 cases, including 4 and 2 cases with HER2-negative and HER2-positive primary tumors, respectively, we conducted repeated measurements of the plasma $H E R 2$ ratios and compared them with clinical courses during the postoperative follow-up period.

At the time of recurrence, plasma HER2 ratios in 7 of 13 patients with HER2-negative primary tumors (53.8\%) were higher than the cutoff value (Table 2), suggesting that the clonal population frequently changed from cells without HER2 amplification to those with HER2 amplification or the tumor frequently acquired HER2 amplification during its progression. Changes in the plasma HER2 ratio during the postoperative follow-up period with the clinical data in four cases (cases 1-4) with recurrence are shown in Fig. 3. In these 4 cases, increases in the plasma HER2 ratio greater than the cutoff value were detected at the time of clinically apparent recurrence, and positive plasma HER2 amplification was continuously observed in all cases during the progression of metastatic tumors, with little or no response to treatments with various chemotherapeutic reagents (Fig. 3). Conventional tumor markers, such as CEA and CA19-9, were less than their cutoff values completely in cases 1 and 2 and partly in cases 3 and 4 during the clinical course. We conducted repeated measurements of the plasma HER2 ratios in one HER2-positive case (case 18) without recurrence as a control experiment (Fig. S3). In this case, plasma HER2 amplification was detected before surgery but plasma $H E R 2$ ratio decreased after surgery and was continuously lower than the cutoff value during the postoperative follow-up periods. These results suggest that plasma $H E R 2$ amplification is a sensitive and reproducible marker for recurrence if the recurrent tumor contains cells with HER2 amplification, and that anti-HER2 therapy may represent an effective reagent for the treatment of these cases.

In four HER2-positive primary tumors, two cases (cases 15 and 17) were negative for a preoperative plasma HER2 ratio (Table 2); case 15 showed an effective response (partial response, PR) to trastuzumab, but it appeared to be minimal because of continuous elevations in the plasma HER2 ratio as well as conventional tumor markers (Fig. 4a). The two cases with plasma HER2 amplification before surgery showed PR to trastuzumab (Table 2). In case 14 with plasma HER2 amplification before surgery, the plasma HER2 ratio increased at the time of recurrence, but clearly and continuously decreased after the treatment with docetaxel plus trastuzumab in accordance with clinically significant efficacy detected by CT and CA19-9 (Fig. 4b).

\section{Discussion}

An important consideration in trastuzumab therapy is temporal and/or spatial heterogeneity, which may change the HER2 status during the clinical course because of genetic differentiation accompanied by neoplastic progression and clonal selection by various factors including the induction of chemotherapy. A previous study on breast cancer showed the frequency of changes in HER2 expression in primary and distant metastatic tumors and 


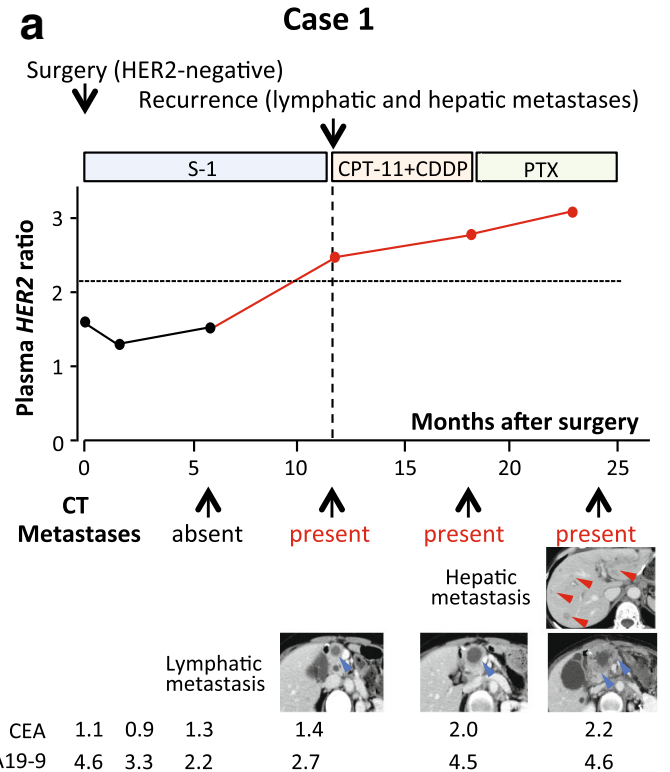

C Case 3

Surgery (HER2-negative)

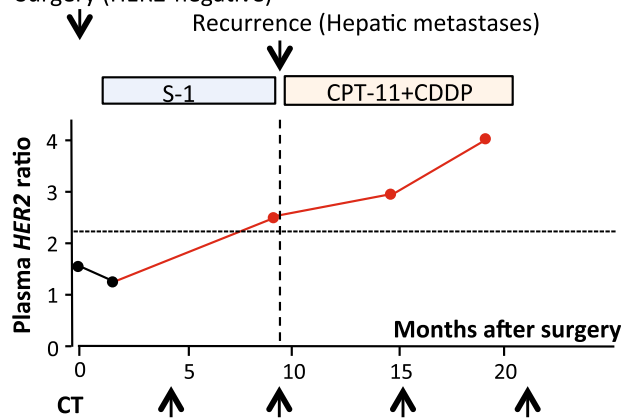

Metastases absent present present present

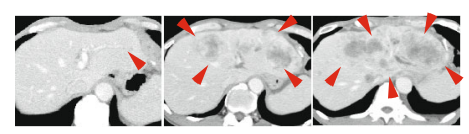

$$
\begin{array}{rrrccc}
\text { CEA } & 1.3 & 1.4 & 2.0 & 4.1 & 71.0 \\
\text { CA19-9 } & 15.6 & 2.0 & 172.9 & 3106.2 & 190353.0
\end{array}
$$

Fig. 3 Changes in plasma HER2 $\mathrm{CN}$ status and other clinical information during clinical courses of GC patients with HER2negative tumors. Cases 1-4 with HER2-negative tumors were treated with anti-cancer drugs other than trastuzumab for postoperative chemotherapy because of their ineligibility for trastuzumab treatments. As increases in their plasma HER2 ratios were observed with recurrence, repeated measurements of the plasma $H E R 2 \mathrm{CN}$ were retrospectively performed during tumor progression. $C D D P$ cisplatin, $C P T-11$ irinotecan, DOC docetaxel, $L N$ lymph node, $S-1$ tegafur, gimeracil, and oteracil potassium combination, $P T X$ paclitaxel, $C E A$ carcinoembryonic antigen, $C A 19-9$ carbohydrate antigen 19-9. a Case

emphasized the importance of confirming the expression status of HER2 in recurrent lesions when considering treatment strategies [22]. However, it is difficult to obtain biopsy samples from recurrent tumors in most GC cases b Case 2
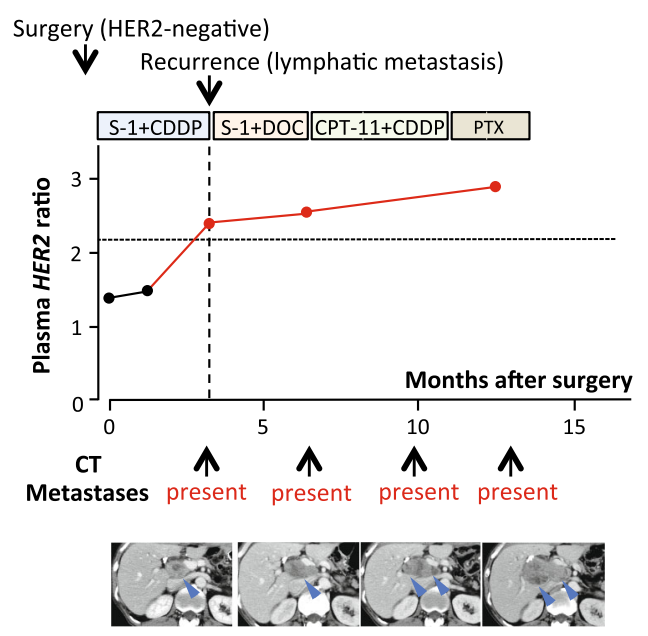

$\begin{array}{rlllll}\text { CEA } & 3.5 & 3.1 & 2.0 & 2.6 & 3.7 \\ \text { CA19-9 } & 11.3 & 9.3 & 7.3 & 12.5 & 6.3\end{array}$

d Case 4

Surgery (HER2-negative)
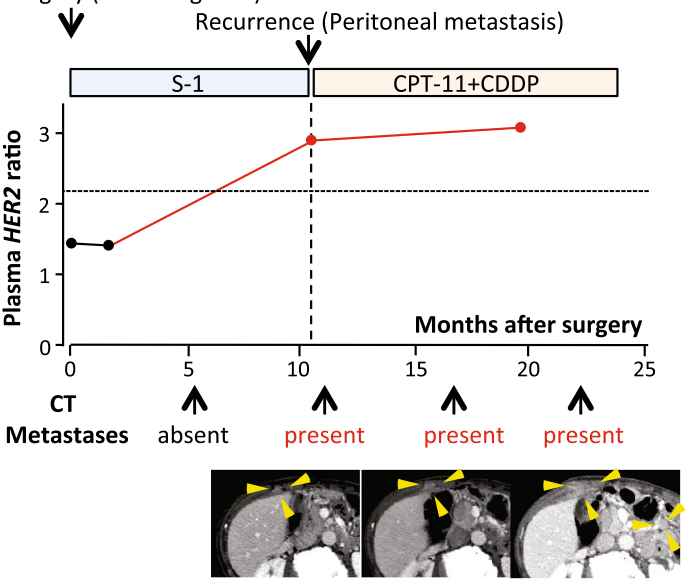

$\begin{array}{ccccc}\text { CEA } & 1.3 & 1.4 & 5.3 & 1.9\end{array}$

$\begin{array}{lllll}\text { CA19-9 } & 2.0 & 5.1 & 11.3 & 6.0\end{array}$

1 is a 63-year-old female patient who underwent surgery for GC with pathological stage T4a, N3, M0, stage IIIC, an IHC HER2 score of 2, and a negative fluorescent in situ hybridization (FISH) result. b Case 2 is a 63-year-old male patient who underwent surgery for GC with pathological stage T2, N1, M0, stage IIA and an IHC HER2 score of 1. c Case 3 is a 64-year-old male patient who underwent surgery for GC with pathological stage T3, N2, M0, stage IIIA, an IHC HER2 score of 2, and a negative FISH result. d Case 4 is a 64-year-old male patient who underwent surgery for GC with pathological stage $\mathrm{T} 4 \mathrm{a}$, $\mathrm{N} 2$, M0, stage IIIB, an IHC HER2 score of 0, and a negative FISH result

because recurrence frequently develops intraabdominally, such as peritoneal or local lymphatic metastases. The plasma HER2 ratio with ddPCR represents a repeatable, noninvasive, and high-throughput analytical approach that 
a

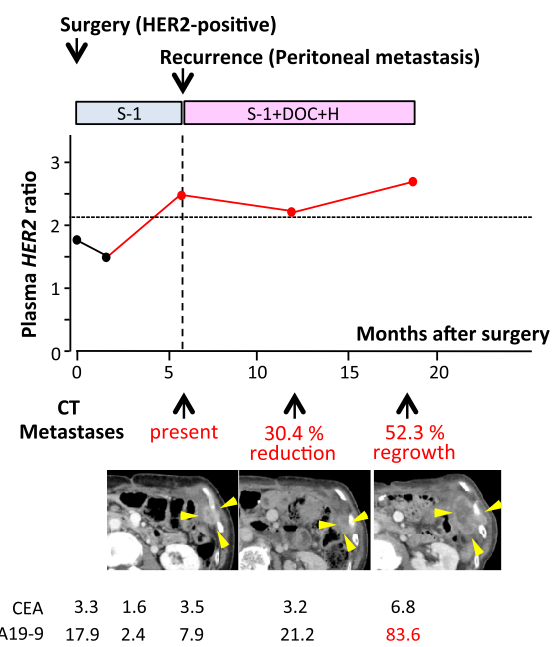

Fig. 4 Changes in plasma $H E R 2 \mathrm{CN}$ status and other clinical information during clinical courses of GC patients with HER2positive tumors. Cases 14 and 15 with HER2-positive tumors were treated with trastuzumab after recurrence because they were not eligible for trastuzumab treatments. $H$ trastuzumab. a Case 15 is a 65-year-old female patient who underwent surgery for GC with pathological stage T4b (liver), N3, M0, stage IIIC, and an IHC HER2 score of 3. An increase in the plasma HER2 ratio was not observed before or immediately after surgery, whereas plasma HER2 amplification was detected after peritoneal recurrence in her abdominal wall. After trastuzumab therapy was initiated, her plasma $H E R 2$ ratio declined and a partial response to trastuzumab therapy was achieved (30.4\% reduction) once. Re-growth of the recurrent tumor was

demonstrates the dynamics of the HER2 status in the clinical courses of GC patients, and may offer an insight to resolve this issue. Plasma HER2 amplification correlated with the therapeutic effects of trastuzumab in patients with HER2-positive tumors, suggesting that the plasma HER2 status is a sensitive marker that reflects responses and resistance to anti-HER2 and other therapies. The positive conversion of plasma HER2 amplification was observed after recurrence in approximately half the patients with HER2-negative tumors, suggesting that a larger number of cases may become new candidates for trastuzumab therapy at recurrence. In these cases, the plasma HER2 status is a useful marker for monitoring therapeutic effects after recurrence.

The present study had several limitations. The small patient population and retrospective nature of the present study do not allow us to draw any concrete conclusions regarding the effectiveness of the ddPCR-based detection of the HER2 CN status of ctDNA in GC patients. Further studies using a larger number of patients and a prospective design are needed. Another limitation is that we had no HER2 status data for recurrent tumors because of difficulties of access, even though stable plasma $H E R 2$ ratios consistent with the results of b

\section{Case 14}

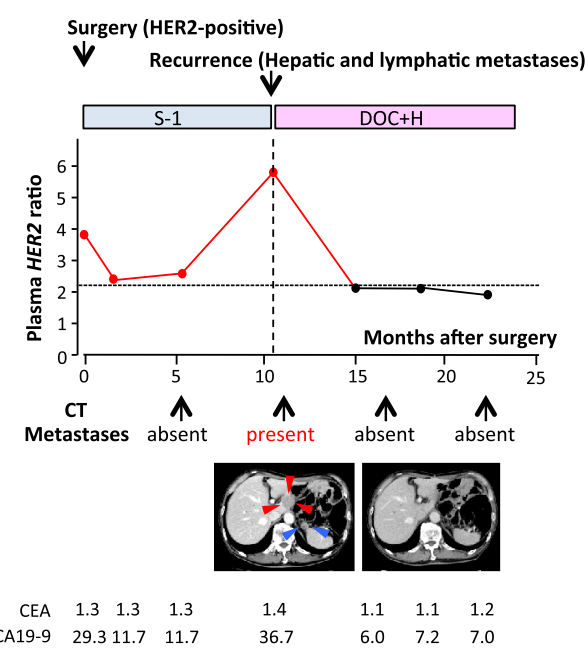

observed 12 months after the initiation of this treatment with a slight elevation in the plasma HER2 ratio. b Case 14 is an 82-year-old male patient who underwent surgery for GC with pathological stage T4a, N2, M0, stage IIIB, and an IHC HER2 score of 3. Although postoperative abdominal hepatic and lymphatic recurrence was detected by computed tomography (CT) 6 months after surgery, a partial response to trastuzumab therapy was achieved 6 months after the initiation of this treatment (disappearance of hepatic and lymphatic metastases). Plasma HER2 amplification was positive before and 1 month after surgery and at the time of recurrence. Notably, a decrease in the plasma HER2 ratio was maintained during the treatment with trastuzumab. CEA and CA19-9 were less than the cutoff values during the whole clinical course

CT and/or other tumor markers were obtained by repeated measurements during the clinical courses of several cases. We also had no direct evidence to show that the heterogeneity of HER2-positive cells affected the results of the plasma HER2 ratio. A comparison of plasma HER2 CN statuses with tumor HER2 statuses determined using samples from multiple sites or the whole resected area as well as recurrent tumors is needed to obtain corroborative data to support our proposal. However, the present study revealed that detection of the plasma HER2 ratio using ddPCR in GC appears to provide an opportunity for reconsidering treatment strategies according to the HER2 status at different times during the clinical course of each patient.

Acknowledgments This work was partially supported by JSPS KAKENHI Grant Number 15K10110 (KO) and 26293304 (II). All authors contributed to the final manuscript.

\section{Compliance with ethical standards}

Conflicts of interest E.O. received research funding from Chugai Pharmaceutical Co., Ltd. (Tokyo, Japan). The funders played no role in the study design, data collection and interpretation, decision to publish, or manuscript preparation. None of the other authors have any conflicts of interest to disclose. 
Ethical statement All procedures followed were in accordance with the ethical standards of the responsible committee on human experimentation (institutional and national) and with the Helsinki Declaration of 1964 and later versions. Informed consent or substitute for it was obtained from all patients for being included in the study.

\section{References}

1. Khalil S, Tan GA, Giri DD, Zhou XK, Howe LR. Activation status of $\mathrm{Wnt} / \beta$-catenin signaling in normal and neoplastic breast tissues: relationship to HER2/neu expression in human and mouse. PLoS One. 2012;7:e33421.

2. Gravalos C, Jimeno A. HER2 in gastric cancer: a new prognostic factor and a novel therapeutic target. Ann Oncol. 2008;19:1523-9.

3. Bang Y-J, Van Cutsem E, Feyereislova A, Chung HC, Shen L, Sawaki A, et al. Trastuzumab in combination with chemotherapy versus chemotherapy alone for treatment of HER2-positive advanced gastric or gastro-oesophageal junction cancer (ToGA): a phase 3, open-label, randomised controlled trial. Lancet. 2010;376:687-97.

4. Nishida Y, Kuwata T, Nitta H, Dennis E, Aizawa M, Kinoshita T, et al. A novel gene-protein assay for evaluating HER2 status in gastric cancer: simultaneous analyses of HER2 protein overexpression and gene amplification reveal intratumoral heterogeneity. Gastric Cancer. 2015;18:458-66.

5. Wang R, Wen H, Xu Y, Chen Q, Luo Y, Lin Y, et al. Circulating microRNAs as a novel class of diagnostic biomarkers in gastrointestinal tumors detection: a meta-analysis based on 42 articles. PLoS One. 2014;9:e113401.

6. Shoda K, Masuda K, Ichikawa D, Arita T, Miyakami Y, Watanabe M, et al. HER2 amplification detected in the circulating DNA of patients with gastric cancer: a retrospective pilot study. Gastric Cancer. 2015;18:698-710.

7. Yasui H, Konishi E, Urasaki K, Yasukawa S, Yanagisawa A. Intratumoral heterogeneity of MIB-1 labelling index in gastric gastrointestinal stromal tumor (GIST). Gastric Cancer. 2006;9:223-8.

8. Lee HE, Park KU, Yoo SB, Nam SK, Park do J, Kim HH, et al. Clinical significance of intratumoral HER2 heterogeneity in gastric cancer. Eur J Cancer. 2013;49:1448-57.

9. Mateo J, Gerlinger M, Rodrigues DN, de Bono JS. The promise of circulating tumor cell analysis in cancer management. Genome Biol. 2014;15:448.
10. Murtaza M, Dawson SJ, Tsui DW, Gale D, Forshew T, Piskorz AM, et al. Non-invasive analysis of acquired resistance to cancer therapy by sequencing of plasma DNA. Nature (Lond). 2013;497:108-12.

11. Lun FM, Chiu RW, Sun K, Leung TY, Jiang P, Chan KC, et al Noninvasive prenatal methylomic analysis by genomewide bisulfite sequencing of maternal plasma DNA. Clin Chem. 2013;59:1583-94.

12. Danese E, Minicozzi AM, Benati M, Montagnana M, Paviati E, Salvagno GL, et al. Comparison of genetic and epigenetic alterations of primary tumors and matched plasma samples in patients with colorectal cancer. PLoS One. 2015;10:e0126417.

13. Flågeng $\mathrm{MH}$, Knappskog $\mathrm{S}$, Haynes $\mathrm{BP}$, Lønning PE, Mellgren G. Inverse regulation of EGFR/HER1 and HER2-4 in normal and malignant human breast tissue. PLoS One. 2013;8:e74618.

14. Whale AS, Huggett JF, Cowen S, Speirs V, Shaw J, Ellison S, et al. Comparison of microfluidic digital PCR and conventional quantitative PCR for measuring copy number variation. Nucleic Acids Res. 2012;40:e82.

15. Hindson BJ, Ness KD, Masquelier DA, Belgrader P, Heredia NJ, Makarewicz AJ, et al. High-throughput droplet digital PCR system for absolute quantitation of DNA copy number. Anal Chem. 2011;83:8604-10.

16. Gevensleben H, Garcia-Murillas I, Graeser MK, Schiavon G, Osin P, Parton M, et al. Noninvasive detection of HER2 amplification with plasma DNA digital PCR. Clin Cancer Res. 2013;19:3276-84.

17. Kinugasa H, Nouso K, Tanaka T, Miyahara K, Morimoto Y, Dohi C, et al. Droplet digital PCR measurement of HER2 in patients with gastric cancer. Br J Cancer. 2015;112:1652-5.

18. Eisenhauer EA, Therasse P, Bogaerts J, Schwartz LH, Sargent D, Ford R, et al. New response evaluation criteria in solid tumours: revised RECIST guideline (version 1.1). Eur J Cancer. 2009;45:228-47.

19. Sobin LH, Wittekind $\mathrm{CH}$, Gospodarowicz M, editors. TNM classification of malignant tumors. 7th ed. New York: Wiley; 2009. p. 73-7.

20. Tomita H, Ichikawa D, Ikoma D, Sai S, Tani N, Ikoma H, et al. Quantification of circulating plasma DNA fragments as tumor markers in patients with esophageal cancer. Anticancer Res. 2007;27:2737-42.

21. Sanders R, Mason DJ, Foy CA, Huggett JF. Evaluation of digital PCR for absolute DNA quantification. PLoS One. 2013;8:e75296.

22. Nakamura R, Yamamoto N, Onai Y, Watanabe Y, Kawana H, Miyazaki M. Importance of confirming HER2 overexpression of recurrence lesion in breast cancer patients. Breast Cancer. 2013;4:336-41. 